\title{
Design strategy and process optimization for reactors with continuous transport of an immobilized enzyme
}

\author{
H.J. Vos and K.Ch.A.M. Luyben \\ Department of Biochemical Engineering, Delft University of Technology Julianalaan 67, 2628 BC Delf (Netherlands) \\ K.R. Westerterp \\ Chemical Reaction Engineering Laboratories, University of Twente, PO Box 217, 7500 AE Enschede (Netherlands)
}

(Received January 18, 1993)

\begin{abstract}
In order to operate a process which uses immobilized enzymes at constant conversion and constant capacity, the refreshment of the enzyme must be continuous. In this paper, two reactor types with continuous refreshment of the biocatalyst are discussed: the stirred tank and the multistage fluidized bed. A method is presented for dimensioning a reactor in such a way that the costs for the conversion of substrate to product are minimized. These costs are calculated as the sum of the biocatalyst consumption and overall reactor costs.

In contrast with the stirred-tank reactor, the multistage fluidized bed can be operated at a non-uniform temperature. For the glucose isomerase process, an optimal temperature gradient results in a small reduction in the biocatalyst consumption $( \pm 5 \%)$. It is concluded that, in general, a temperature gradient will only favour the economy of processes with relatively expensive biocatalysts.

Compared with conventional reactor types, such as the continuous stirred-tank reactor and the fixedbed reactor, the multistage fluidized-bed reactor can improve the economy of an enzyme-catalysed reaction significantly.
\end{abstract}

\section{Introduction}

Enzymes show a relatively high activity at moderate temperatures and they are often stereospecific and/or regiospecific in their catalytic activity. A disadvantage is, however, their sensitivity to environmental conditions. They are easily poisoned by, for example, metal ions and are only active over a limited range of temperatures and $\mathrm{pH}$ values. It is therefore essential that processes using enzymes as biocatalysts are operated at well-defined and carefully chosen conditions.

The glucose isomerase process described by Oestergaard and Knudsen [1] provides a good example of an enzyme dependent process. A glucose syrup, which is produced from corn starch, is partly converted into a 42 wt.\% fructose syrup by immobilized glucose isomerase. The sweetening ability of this high fructose corn syrup (HFCS) is approximately equal to that of sucrose [2]. Insoluble impurities in the influent glucose syrup, such as wax and proteins, are removed by filtration. Soluble impurities, such as peptides, amino acids and salts, are removed by treatment with active carbon followed by ion exchange. After these purification steps, the glucose syrup enters the reactor section for isomerization. A long syrup residence time will cause byproduct formation and a $\mathrm{pH}$ decrease. In order to minimize the formation of byproducts, and also because the $\mathrm{pH}$ influences both the activity and the stability of the enzyme, a short residence time in the reactor section is thus important. Therefore, as with most reactions involving immobilized enzymes, fixed-bed reactors are generally used. An additional problem is that, while the reactor temperature must be high enough to prevent microbial contamination, a lower temperature would favour the cumulative productivity that can be obtained with the enzyme. At a high temperature the biocatalyst is less stable, but, because it is more active, a smaller reactor volume is needed. Therefore there is an optimization problem.

Regardless of the reactor temperature, the enzyme in the reactor will have lost most of its activity after a certain operating time and must be replaced. As it is desirable to maintain a constant conversion, 
this means that continuous adjustment of the substrate flow rate is nccessary. In order to reduce these flow adjustments, a configuration of reactors in series and parallel is often used and the biocatalyst is changed sequentially. Nevertheless, a configuration of six reactors still results in fluctuations of $\pm 10 \%$ of the average flow rate [1]. The substrate solution flows through the reactors in the direction of increasing enzyme activity. This allows any harmful contaminants still present in the glucose syrup to adsorb on the more deactivated biocatalyst, protecting the fresh enzyme from poisoning reactions.

In this paper, we describe the design and optimization of an alternative to this type of process. Central to this new development is a multistage fluidized-bed reactor (MFBR) with continuous refreshment of the deactivating biocatalyst which has been described in detail by Vos et al. [3]. The biocatalyst is transported from the top of the reactor downwards through holes in distributor plates by periodic inversion of the normally upward liquid flow. Frequent transport of small quantities results in a constant biocatalyst hold-up in each reactor compartment (Fig. 1). Compared with a series of fixed-bed reactors, the advantage of this system is that it can be operated at a constant flow rate and conversion. An additional advantage is the reduced danger of fouling and, in the case of gel immobilization, the circumvention of the risk of a high pressure drop over the reactor which can cause compression and deformation of the biocatalyst particles [4]. Furthermore, the addition and removal of biocatalyst particles are continuous and automatic. A possible disadvantage of the MFBR is the requirement of biocatalyst particles with a more or less uniform diameter and density [5]. For reactions that require continuous $\mathrm{pH}$ control to compensate for a possible $\mathrm{pH}$ change due to the reaction, such

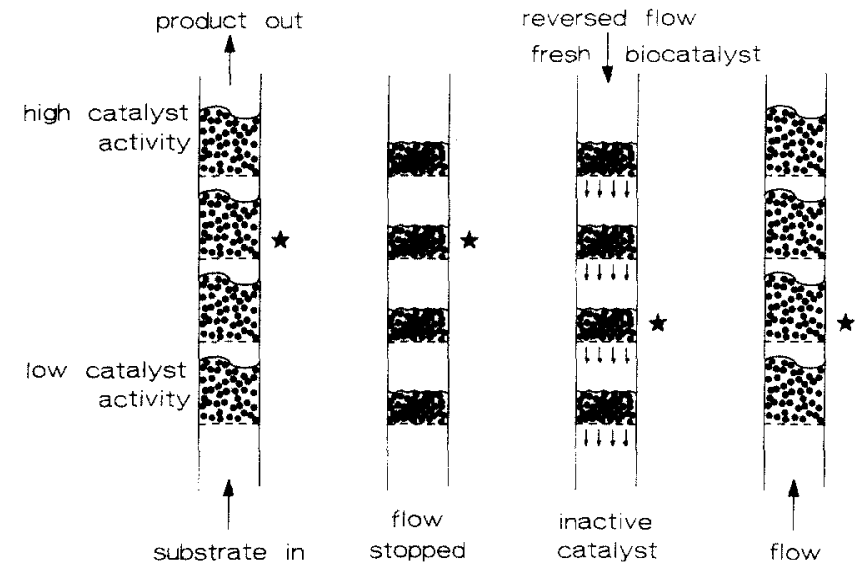

Fig. 1. Counter-current MFBR for use with immobilized enzymes, with frictional refreshment of enzymes. as the production of 6-amino-penicillanic acid (6APA) [6], a series of well-mixed reactors might be preferred to an MFBR with a plug flow characteristic.

As already mentioned, the operating temperature of the reactor influences the activity and the stability of the immobilized biocatalyst, and thus also the size of the reactor. Therefore process optimization and reactor design have to be studied simultaneously. During previous work on the optimal temperature control policy for enzyme-catalysed reactions in a batch reactor, it was shown that small savings in biocatalyst consumption could be attained by increasing the temperature at the end of the reaction time [7]. For immobilized glucose isomerase in a fixed-bed reactor, it was shown that, if the reactor temperature was increased with the aging of the biocatalyst, savings of up to more than $10 \%$ are possible [8]. For the MFBR this implies that a somewhat higher temperature in the bottom compartments would be beneficial. This could, for example, be realized by a relatively high temperature of the influent. As the reactor loses heat to the surroundings, this would result in a temperature gradient as the liquid passes up the reactor.

The aim of this work is to determine the optimal process conditions for reactors using continuous refreshment of a biocatalyst which gradually becomes deactivated, in particular the MFBR. Optimization implies the search for those values of the process optimization variables that result in an extreme (i.e. maximum or minimum) of some process property which is defined by an objective function. Since the production of chemicals is an economic activity, the discounted cash flow rate of return (DCFRR) is the best property to be maximized. The DCFRR is the interest rate at which the net present value of the process is zero. Thus expenses or earnings at the beginning of the project weigh more heavily than later cash flows. Because uncertainties such as price fluctuations during the entire life time of the process are involved in this method, costs minimization for studies during the design phase is generally regarded as a good alternative to the DCFRR method [9].

In order to relate costs functions to processes which use enzymes which gradually become deactivated, we start with the presentation of a model for the MFBR which has been described more extensively elsewhere [3] and verified with experiments using immobilized glucose isomerase [10]. In order to illustrate the use of this generally applicable optimization strategy, its application for the continuous stirred-tank reactor (CSTR) will be discussed. Based on a simplified model, an analytical expression for the optimal CSTR temperature is 
derived. For the MFBR, the problem is more complex and a numerical method for design and process optimization is presented. The minimization of the costs for the production of HFCS is also illustrated.

\section{Model for the multistage fluidized-bed reactor}

To describe the deactivation of the biocatalyst, a first-order model is used. This is often sufficiently accurate to describe the enzyme deactivation kinetics [11]. The biocatalyst is immobilized in spherical particles of uniform composition. Furthermore, the reactor is assumed to operate at a constant pH, while the temperature is taken as a variable in order to optimize the reaction conditions. The relationship between temperature and enzyme activity can be described by the Arrhenius equation

$k_{\mathrm{r}}^{\prime}(T)=\omega k_{\mathrm{r}}(T)=\omega k_{\mathrm{r} 0} \exp \left(\frac{-E_{\mathrm{r}}}{R T}\right)$

In this equation, $\omega$ is the fraction of the initial amount of enzyme that is still active. The first-order deactivation is based on a one-step mechanism with the assumption that the biocatalyst can only exist in two different states, active and inactive:

$E_{\text {active }} \longrightarrow E_{\text {inactive }}$

The first-order deactivation rate constant of this one-step deactivation reaction is described as follows:

$k_{\mathrm{d}}(T)=K_{\mathrm{d} 0} \exp \left(\frac{-E_{\mathrm{d}}}{R T}\right)$

The biocatalyst activity in each reactor compartment can be determined from the assumptions that there is perfect particle mixing in each compartment and that the mean biocatalyst activity in a compartment can be described by the activity corresponding to particles with an age equal to the mean residence time of the particles in that compartment. In general, this is a simplification of the real situation where particles of different ages, and thus different activities, are present in a reactor compartment. However, for first-order deactivation, the segregation of biocatalytic activity does not influence the mean value of biocatalyst activity in the compartments. Thus, for a reactor with $N$ compartments numbered from $n=1$ at the bottom to $n=N$ at the top, first-order deactivation and a total biocatalyst residence time of $\tau_{b}$ in the reactor, the fraction of active biocatalyst in a compartment $n$ is given by
$\omega_{n}=\prod_{i=n}^{N}\left(1+\frac{\tau_{\mathrm{b}}}{N} k_{\mathrm{d}}\left(T_{i}\right)\right)^{-1}$

For this equation, a uniform temperature in every reactor compartment has been assumed.

For the substrate, radial gradients are assumed to be absent. The transport of the liquid phase through each reactor compartment is described by plug flow with axial dispersion. A four-tanks-inseries model is suitable for quantifying the axial dispersion in a compartment of the MFBR [10, 12]. For $m$ liquid mixing zones per reactor compartment, the following balance holds for such a mixing zone:

$c_{\mathrm{L}, \mathrm{in}}-c_{\mathrm{L}}=\frac{\left(\mathrm{Da}_{1}\right)_{n}}{m} \eta_{\mathrm{ov}} \nu_{\mathrm{L}}$

The reactor model is solved by calculation of the $\mathrm{Da}_{1}$ number for every compartment using eqn. (4), followed by solving the $m N$ balances for the liquid mixing zones starting at the bottom of the reactor. Expressions for the dimensionless bulk concentration $c_{\mathrm{L}}$, the dimensionless concentration dependence $\nu_{\mathrm{L}}$ of the reaction rate and the $\mathrm{Da}_{1}$ number, which represents the ratio of the maximum reaction rate to that convective transport, are given in Table 1 for various kinetic rate equations. The overall effectiveness factor $\eta_{\text {ov }}$ accounts for the decrease in reaction rate inside the particle due to external mass transfer resistance and diffusion limitation. The overall effectiveness factor is dependent of $\omega$, and $\eta_{\text {ov }}$ does not exhibit a first-order dependence on the enzyme age. Therefore the mean value of the effectiveness factor in a reactor compartment is influenced by the fact that not all particles have the same age and thus the same activity. This is not incorporated in eqn. (5), but the inaccuracy introduced by not taking the segregation of biocatalytic activity into account in the calculation of the mean value of the effectiveness factor is reduced with an increase in the number of reactor compartments. Various methods for calculating the ef-

TABLE 1. Dimensionless numbers for various kinetic rate equations

\begin{tabular}{llll}
\hline Kinetics & $(\mathrm{Da})_{n}$ & $\nu_{\mathrm{L}}$ & $c_{\mathrm{L}}$ \\
\hline Michaelis-Menten & $\frac{(1-\epsilon) H V_{\max }}{u_{0} K_{\mathrm{M}}} \omega_{n}$ & $\frac{c_{\mathrm{L}}}{1+c_{\mathrm{L}}}$ & $\frac{S_{\mathrm{L}}}{K_{\mathrm{M}}}$ \\
Zero order & $\frac{(1-\epsilon) H k_{0}}{u_{0} S^{0}} \omega_{n}$ & 1 & $\frac{S_{\mathrm{L}}}{S^{0}}$ \\
Reversible first-order & $\frac{(1-\epsilon) H k_{1}}{u_{0}} \omega_{n}$ & $c_{\mathrm{L}}-c_{\mathrm{L}, \mathrm{eq}}$ & $\frac{S_{\mathrm{L}}}{S^{0}}$ \\
& & &
\end{tabular}


fectiveness factor for enzyme kinetics have been discussed and compared by Vos et al. [13].

\section{Process optimization strategy}

The process which is the subject of this optimization study involves a reaction which is catalysed by an immobilized enzyme. It might be part of a larger continuous production plant, and thus the process is characterized by a specified capacity and feed composition. The degree of conversion is dictated by the desired quality of the product stream.

In order to optimize the reactor design, the total costs function can be minimized. A general costs function of a chemical process is as follows [9]:

$\dot{C}_{\mathrm{TOT}}=\dot{C}_{\mathrm{RM}}+\dot{C}_{\mathrm{DISP}}+\dot{C}_{\mathrm{VAR}}+\dot{C}_{\mathrm{FIX}}$

The total costs per unit of time consist of costs $\dot{C}_{\mathrm{RM}}$ for raw materials, costs $\dot{C}_{\mathrm{DISP}}$ for disposal of waste materials, and variable costs $\dot{C}_{\mathrm{VAR}}$ and fixed costs $\dot{C}_{\mathrm{FTX}}$. In this particular case, the flow rate and compositions of the reactor influent and effluent are fixed; thus, when minimizing the total costs of the process, only the variable and fixed costs need to be taken into account. The fixed costs, or overall reactor costs, can be subdivided into truly fixed costs (e.g. personnel) and investment-related fixed costs (e.g. insurance, maintenance and depreciation costs). For this process, the variable costs are mainly determined by biocatalyst consumption and energy requirements. However, biocatalysts are only active and stable in a narrow temperature range; thus the energy requirements can be regarded as not affected by the optimal choice for the reactor temperature. By doing so, the optimization problem can be separated into technological and economic problems. The technological aspect is the determination of the operating temperature at a given biocatalyst residence time, substrate flow and conversion, such that the hold-up of biocatalyst (i.e. the total amount of biocatalyst present in the reactor) is minimized. This simultaneously minimizes the biocatalyst consumption (variable costs) and the reactor dimensions (fixed costs), and thus also the total costs function. The economic aspect is to make an optimum selection from the combinations of biocatalyst residence time and operating temperature that can be regarded as technological suboptima. This is easily illustrated for a continuous stirred-tank reactor (CSTR) with continuous replacement of the enzyme.

\section{Illustration of the optimization strategy for a continuous stirred-tank reactor}

The three most important process variables which determine the size of a CSTR, and thus the investment-related fixed costs, are the residence time, the hold-up of biocatalyst and the operating temperature. Analogous to the model of the MFBR, it is assumed that the performance of the well-mixed biocatalyst particles can be described by the activity and effectiveness factor equal to those of particles with an age equal to the mean residence time of the biocatalyst. For a single stirred reactor, this might be a somewhat rough approach, but it can be used quite well to illustrate the optimization strategy. As a result, the overall reaction rate constant, in the case of first-order deactivation, is given by the following equation:

$k_{\mathrm{r}}{ }^{\prime}=k_{\mathrm{r}} \omega=\frac{k_{\mathrm{r}}(T)}{1+\tau_{\mathrm{b}} k_{\mathrm{d}}(T)}$

For given values of $\tau_{\mathrm{b}}$ and $T$, the hold-up of biocatalyst follows from $k_{\mathrm{r}}{ }^{\prime}$ and the desired conversion; thus only two of the three process design variables can be chosen freely. From eqn. (7), the effect of temperature on the overall reaction rate constant for various biocatalyst residence times is shown in Fig. 2. Arbitrary parameter values have been used together with first-order reaction kinetics. The maximum attainable activity is higher for short biocatalyst residence times. A high temperature causes rapid deactivation and thus a low mean activity; at low temperatures the deactivation reaction is very slow and the activity is almost independent of the biocatalyst residence time.

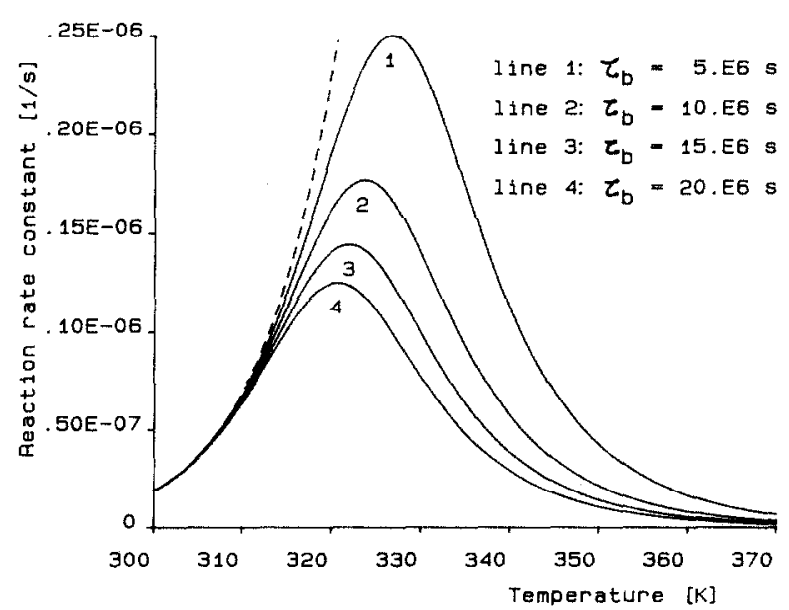

Fig. 2. First-order reaction rate constant as a function of temperature for various biocatalyst residence times in a CSTR $(-)$ and the reaction rate constant without deactivation $(---)$ $\left(k_{\mathrm{r} 0}=5 \times 10^{9} \mathrm{~s}^{-1} ; k_{\mathrm{d} 0}=2 \times 10^{25} \mathrm{~s}^{-1} ; E_{\mathrm{r}}=100 \mathrm{~kJ} \mathrm{~mol}^{-1} ; E_{\mathrm{d}}=200\right.$ $\mathrm{kJ} \mathrm{mol}^{-1}$ ). 
Now we shall focus on the technological aspect of the optimization problem: the determination of combinations of $\tau_{b}$ and $T$ for which the hold-up of biocatalyst is minimal. Thus the reaction rate per unit of biocatalyst is maximal. Maximization of the overall reaction rate constant results in a maximal reaction rate per unit of biocatalyst. Possible mass transfer resistances related to heterogeneous catalysis do not influence the optimum temperature of the CSTR. The optimal combinations of $\tau_{\mathrm{b}}$ and $T$ are therefore found by simply making the derivative of the reaction rate constant with respect to temperature equal to zero:

$\frac{\partial}{\partial \mathrm{T}}\left(\frac{k_{\mathrm{r} 0} \exp \left(-E_{\mathrm{r}} / R T\right)}{1-\tau_{\mathrm{b}} k_{\mathrm{d} 0} \exp \left(-E_{\mathrm{d}} / R T\right)}\right)=0$

Thus

$\frac{1}{T_{\mathrm{opt}}}=\frac{R}{E_{\mathrm{d}}} \ln \left[k_{\mathrm{d} 0} \tau_{\mathrm{b}}\left(\frac{E_{\mathrm{d}}}{E_{\mathrm{r}}}-1\right)\right]$

From this, it follows that an optimal operating temperature for a given biocatalyst residence time exists for $E_{\mathrm{d}}>E_{\mathrm{r}}$. In principle, the same approach as illustrated for the CSTR can be used for the MFBR.

\section{Application of the optimization strategy to the multistage fixed-bed reactor}

Compared with the CSTR, the MFBR is a more complex reactor with more design parameters. However, this does not necessarily increase the complexity of the optimization problem. These extra parameters are determined by some conditions that are essential for successful operation of the MFBR, as will be discussed briefly.

(1) Within a batch of immobilized biocatalyst particles, there always will be a certain spread in size. When particle mixing is incomplete, the smaller particles tend to accumulate in the top of a compartment and, since the biocatalyst is mainly transported from the bottom of a compartment, this causes an unequal residence time for small and large particles. Therefore for an efficient use of the biocatalyst it is essential that the particles are perfectly mixed. The conditions under which particles can be mixed are dependent on the properties of the particles and liquid, and the scale of the reactor [5]. These conditions must be determined from fullscale experiments and can be related to the bed porosity, e.g. $0.6 \leqslant \epsilon \leqslant 0.7$. As a result, the required liquid velocity follows from the particle properties (diameter and density) and the demand for the bed porosity. The Richardson-Zaki [14] equation is often used for this purpose.

(2) The diameter of the reactor is determined by the allowable liquid velocity and the desired capacity.

(3) For a single CSTR, particles with high and low activities are simultaneously removed from the reactor. For an MFBR, approximately plug flow transport of the biocatalyst can be realized by increasing the number of reactor compartments, and only particles with a low activity are removed from the reactor. The reactor efficiency improves with increasing number of compartments but requires a larger investment for extra distributor plates. It is not expected that this is a major cost factor in the overall process economics, and we therefore assume, somewhat arbitrarily, that ten compartments are used in order to achieve a limited degree of back mixing of the biocatalyst. If desired, the number of plates can be optimized by comparing the optimization results for different numbers of compartments.

The resulting procedure for the optimal design of an MFBR is summarized in Fig. 3. For given values of the compartment temperatures, the biocatalyst residence time and the number of reactor compartments, the active biocatalyst fraction $\omega_{1}$, $\omega_{N}$ in each reactor compartment can be calculated from eqn. (4). From the temperature and the biocatalyst activity in the compartments, the liquid residence time is found iteratively, such that the desired degree of conversion is attained. The bed height, which is proportional to the biocatalyst loading, is calculated from the liquid residence time and the liquid velocity. Because of a biocatalyst activity gradient and a possible temperature gradient, the effectiveness factor is different for every reactor compartment. Therefore the mass transfer resis-

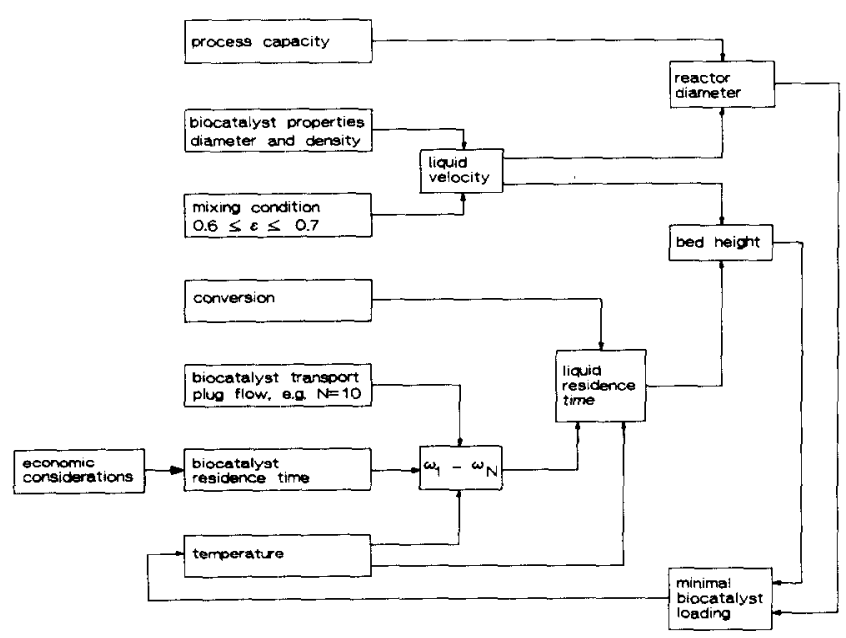

Fig. 3. Diagram for the optimal design of an MFBR. 
tances related to heterogeneous catalysis do influence the optimal operating conditions of the MFBR, unlike those of the CSTR.

The technological aspect of the optimization problem is the determination of the temperatures in the reactor compartments at a given value of the biocatalyst residence time, such that the hold-up of biocatalyst is minimized. A non-linear parameter estimation routine must be used to find these temperatures. From the technologically optimal combinations of temperature and biocatalyst residence time which can be obtained by following this procedure, the economically optimal combination can be selected by minimization of a total costs function.

\section{A case study: optimal design of a multistage fixed-bed reactor for the production of high fructose corn syrup}

As an example, a case study for the optimization of the glucose isomerase process in an MFBR is presented. A typical capacity for an HFCS production unit is 100 ton day ${ }^{-1}$ dry weight [1]. The feed and product stream compositions have been taken from published data [2] and are listed in Table 2. Like most enzymatic reactions, heat effects can be neglected. The reaction rate can be dcscribed by reversible first-order kinetics according to

$v(S)=k_{1}\left(S-S_{\text {eq }}\right)$

where

$S_{\mathrm{eq}}=\frac{S+P}{1+K_{\mathrm{eq}}}$

In general, numerical techniques must be used for calculation of the effectiveness factor for reversible reaction kinetics. Only for irreversible zero- and first-order reactions have analytical solutions been derived. However, when $S_{\text {eq }}$ is a constant within the biocatalyst particle, the differential equation and boundary conditions that describe the concentration profile in the catalyst pellet are mathematically equivalent to the differential equation for irreversible

TABLE 2. Feed and product stream compositions for the high fructose corn syrup process

\begin{tabular}{lll}
\hline & $\begin{array}{l}\text { Inlet } \\
\text { (\% of dry weight) }\end{array}$ & $\begin{array}{l}\text { Outlet } \\
\text { (\% of dry weight) }\end{array}$ \\
\hline Dry weight & 45 & 45 \\
Glucose & 95 & 53 \\
Fructose & 0 & 42 \\
Oligosaccharides & 5 & 5 \\
\hline
\end{tabular}

first-order kinetics. The effectiveness factor follows from the solution of this differential equation; thus by assuming that $S_{\text {eq }}$ is constant the first-order effectiveness factor expression can be applied. Because the mass transport properties of $S$ and $P$ are similar, their spatial concentration curves are almost symmetrical and this results in a nearly constant value of $S+P$ and therefore, from eqn. (11), in a constant value of $S_{\text {eq. }}$. Therefore, eqn. (12), which is the expression for the overall effectiveness factor for first-order kinetics [13], can be applied for this equilibrium reaction:

$\eta_{\mathrm{ov}}=\left(\frac{3 \Phi^{2}}{3 \Phi \operatorname{coth}(3 \Phi)-1}+\frac{3 \Phi^{2}}{\mathrm{Bi}}\right)^{-1}$

For the model biocatalyst, Maxazyme GI Immob of Gist-brocades n.v. was selected. The kinetic and physical properties of the spherical brown particles have been taken from Roels and van Tilburg [15] and are listed in Table 3 . It should be noted that the effective diffusivity is not given as a function of temperature. The relationship between the temperature and the reaction rate constant has been determined assuming a constant effective diffusivity; thus the influence of the temperature on the effective diffusivity is incorporated in the reaction rate constant. This will not introduce large inaccuracies because, in the narrow temperature range of interest, the variation in the diffusivity with the temperature is small compared with the variation in the reaction rate constant.

The viscosity of a syrup with $45 \%$ dry weight was correlated as a function of the temperature by interpolation of published data [16], the density is taken from the Handbook of Chemistry and Physics [17].

The particle density and fluidization behaviour were analysed in a tubular reactor with an internal diameter of $0.02 \mathrm{~m}$ which was thermostated at 333 $\mathrm{K}$. The initial particle density was approximately $1100 \mathrm{~kg} \mathrm{~m}^{-3}$, but this increased when the particles were soaked with syrup and fluidization was possible. The liquid fraction was determined at various liquid velocities and fitted with the Richardson-Zaki

TABLE 3. Physical and kinetic data relevant to the high fructose corn syrup process

\begin{tabular}{ll}
\hline$k_{1}$ & $4.83 \times 10^{9} \exp (-9500 / T) \mathrm{s}^{-1}$ \\
$k_{\mathrm{d}}$ & $1.75 \times 10^{25} \exp (-24400 / T) \mathrm{s}^{-1}$ \\
$D_{\mathrm{eff}}$ & $6.7 \times 10^{-11} \mathrm{~m}^{2} \mathrm{~s}^{-1}$ \\
$R_{\mathrm{p}}$ & $6 \times 10^{-4} \mathrm{~m}$ \\
$K_{\mathrm{eq}}$ & $28.8 \exp (-1100 / T)$ \\
$\mu$ & $14.44 \times 10^{-3} \exp [-0.04(T-273)]+0.90 \times 10^{-3} \mathrm{Pas}$ \\
$\rho_{\mathrm{L}}$ & $1195 \mathrm{~kg} \mathrm{~m}^{-3}$ \\
\hline
\end{tabular}


equation

$\frac{u_{0}}{u_{\mathrm{t}}}=\epsilon^{n \mathrm{Rz}}$

The results are shown in Fig. 4. The exponent, $n_{\mathrm{RZ}}$ was determined to be 2.9 and, from the terminal velocity, the particle density was calculated at 1210 $\mathrm{kg} \mathrm{m}^{-3}$.

The external mass transfer coefficient was calculated from a correlation by Tournié et al. [18]. The values obtained for the $\mathrm{Bi}$ number resulted in an external effectiveness factor of approximately 0.96 ; thus the external mass transfer resistance only has a small effect on the reaction rate.

Now that we have determined all the process parameters, it is possible to simulate reactor performance. A normal operational lifetime for immobilized glucose isomerase is 50 days [19]. For this biocatalyst residence time, we compared several approaches for the determination of the optimal operating temperature. The reactor temperature can be treated as constant or it can be described by a polynomial $\left(N_{\mathrm{p}} \geqslant 2\right)$ :

$T_{n}=p_{1}+\sum_{i=2}^{N_{p}} p_{i}(N-n)^{i-1}$

Alternatively, we could have used an optimization method involving all the compartment temperatures. The advantage of the approach used here is that a ten-compartment reactor can be optimized rather accurately using only a few parameters. From the various hill-climbing routines that have been developed for numerical parameter optimization in a multidimensional space, the direct grid search [20] was applied.

Up to $N_{p}=4$, the parameter values $p_{1}, p_{2}, p_{3}$ and $p_{4}$ were calculated such that the hold-up of biocatalyst in the reactor was minimized. The optimal

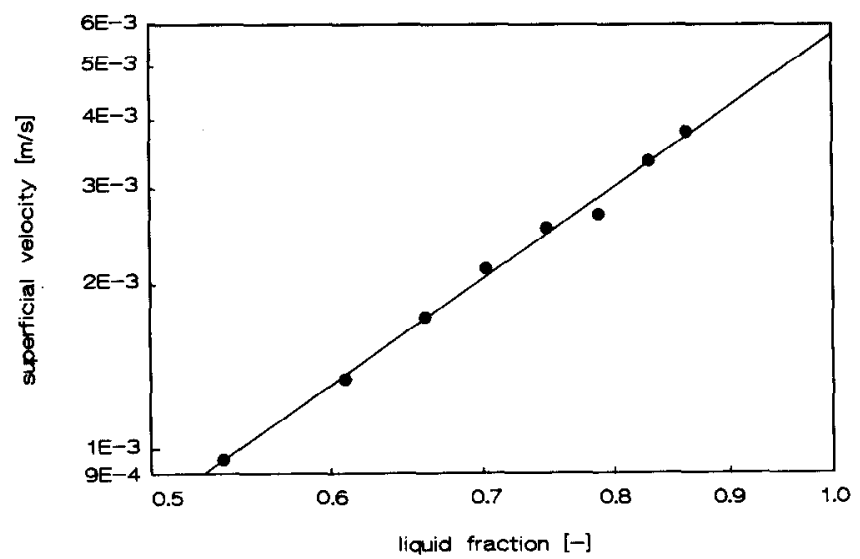

Fig. 4. Superficial liquid velocity and bed porosity for immobilized glucose isomerase in 45 wt.\% glucose solution. temperature was also calculated from eqn. (9). The results are listed in Table 4. Figure 5 gives a graphical representation of various parameters in the isomerization process for $N_{\mathrm{p}}=4$.

Comparing the biocatalyst consumption values obtained with the various optimization methods used, it appears that eqn. (9) gives a reasonable approximation of the optimum when using a uniform reactor temperature. The savings in biocatalyst consumption that can be obtained by imposing a temperature gradient on the reactor are relatively small and of the order of magnitude of $5 \%$.

Estimating the cost of the biocatalyst as US\$9 $\mathrm{kg}^{-1}$ and the market price of HFCS as US $\$ 0.5 \mathrm{~kg}^{-1}$ dry weight [21], a reduction of the biocatalyst consumption of $5 \%$ results in a capital saving of, at most, US\$22 000 year $^{-1}$ which is equivalent to approximately $0.12 \%$ of the price of the syrup produced. This must be compared with the cost of controlling the temperature of the reactor, such that the calculated temperature profile (Fig. 5) is maintained. The residence time of the syrup in the reactor is approximately $2 \mathrm{~h}$; thus the superficial liquid velocity is less than $2 \times 10^{-3} \mathrm{~m} \mathrm{~s}^{-1}$. This results in a low heat transfer coefficient and, even for a wall temperature of $273 \mathrm{~K}$, the cooling capacity is too low to achieve the desired temperature gradient of approximately $10 \mathrm{~K}$. Thus, in order to operate this process with minimal biocatalyst requirements, additional investments have to be made for internal cooling devices, or the process should be carried out in a several parallel reactors with wall cooling. Taking this into consideration, it is expected that for isomerization in an MFBR, a reactor without thermal insulation is the best choice, taking advantage of the small temperature gradient caused by cooling against the surroundings.

For the HFCS process in an MFBR at constant temperature, the technological suboptima were determined for various biocatalyst residence times (Fig. 6). When the biocatalyst residence time is increased, the optimum reactor temperature decreases. To compensate for this, the biocatalyst hold-up per compartment is increased. The residual activity $\omega_{1}$ in the bottom compartment is more or less constant, at approximately $40 \%$. Intuitively, one would possibly think that, in order to obtain maximum productivity per unit of biocatalyst, it is advantageous to use the biocatalyst down to a low residual activity. However, the optimum is determined by two factors that act in opposite directions. Although the optimum lies at $40 \%$ residual activity, the biocatalyst can be used to, for example, $25 \%$ residual activity. This requires a higher reactor temperature, resulting in a higher reaction rate 


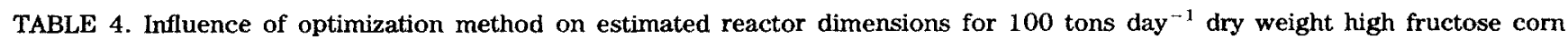
syrup production in a multistage fluidized-bed reactor (mass flow, $2.572 \mathrm{~kg} \mathrm{~s}^{-1}$; biocatalyst residence time, $4.32 \times 10^{6} \mathrm{~s}(=50$ days); $m=4$; bed porosity, $0.65 ; N=10 ; S_{0}=513 \mathrm{~kg} \mathrm{~m}{ }^{-3} ; \xi=0.4421$ )

\begin{tabular}{|c|c|c|c|c|c|}
\hline \multirow[t]{2}{*}{ Optimization method } & \multicolumn{5}{|l|}{ Value } \\
\hline & Equation (9) & $N_{\mathrm{p}}=1$ & $N_{\mathrm{p}}=2$ & $N_{\mathrm{p}}=3$ & $N_{\mathrm{p}}=4$ \\
\hline Biocatalyst per reactor compartment $(\mathrm{kg})$ & 679.1 & 669.3 & 644.8 & 639.8 & 639.3 \\
\hline Compartment height $(\mathrm{m})$ & 1.39 & 1.37 & 1.32 & 1.31 & 1.31 \\
\hline Reactor diameter $(\mathrm{m})$ & 1.21 & 1.21 & 1.21 & 1.21 & 1.21 \\
\hline Top temperature $(\mathrm{K})$ & 330.8 & 332.3 & 328.7 & 330.3 & 330.3 \\
\hline Bottom temperature $(\mathrm{K})$ & 330.8 & 332.3 & 337.4 & 339.9 & 340.5 \\
\hline Residual activity $\omega_{1}$ & 0.511 & 0.398 & 0.283 & 0.247 & 0.239 \\
\hline Biocatalyst consumption relative to $N_{\mathrm{p}}=1$ (\%) & 101.5 & 100.0 & 96.3 & 95.6 & 95.5 \\
\hline
\end{tabular}

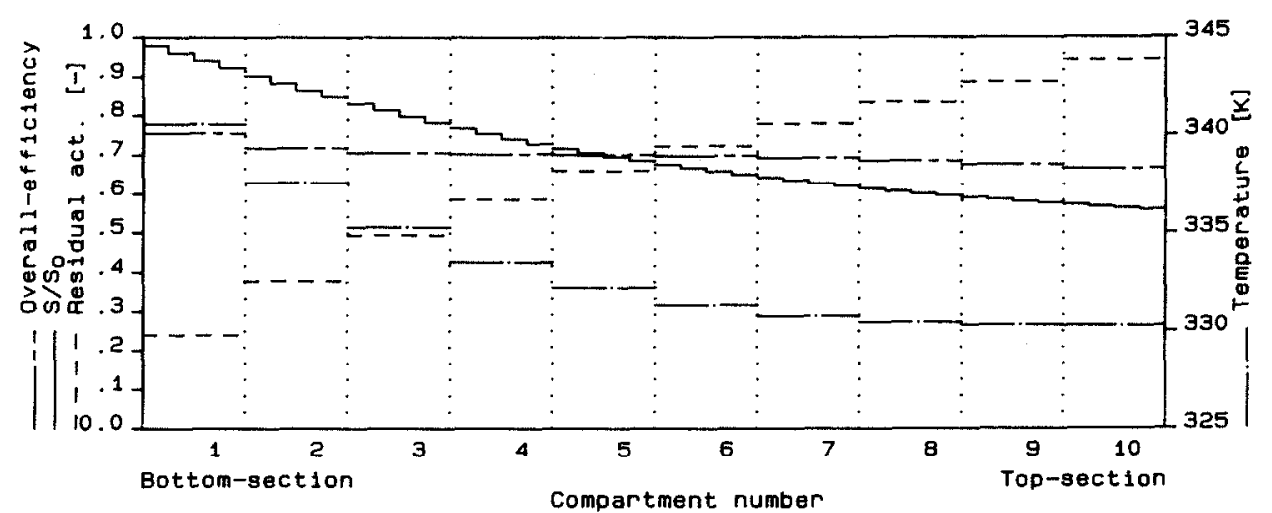

Fig. 5. Glucose concentration, biocatalyst activity, overall effectiveness factor and reactor temperature for the HFCS process (parameters as in Table $4 ; N_{\mathrm{p}}=4$ ).

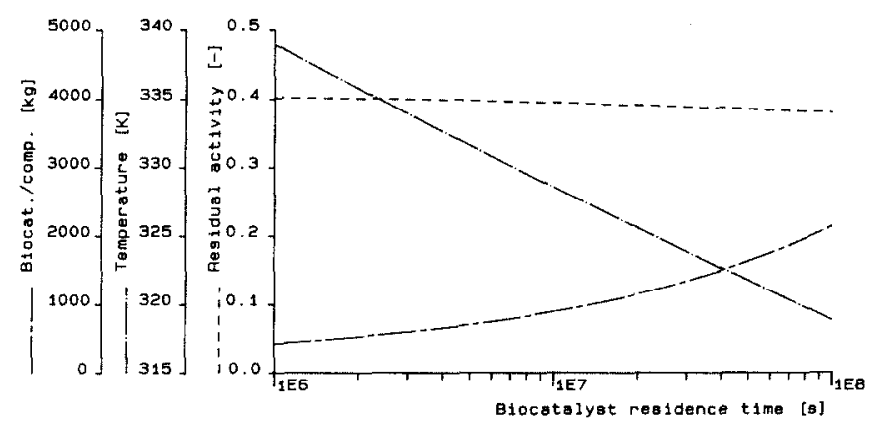

Fig. 6. Residual biocatalyst activity $\omega_{1}$, reactor temperature and biocatalyst hold-up for 100 tons day ${ }^{-1}$ dry weight HFCS reactor: optimization for $N_{p}=1$ as a function of the biocatalyst residence time.

constant (eqn. (1)), but the total hold-up of active biocatalyst is lower.

The glucose isomerase biocatalyst considered here must be used to approximately $40 \%$ residual activity, regardless of the biocatalyst residence time. When taking into account the temperature restrictions, in order to keep the process free from microbial contamination, the picture changes drastically (Fig. 7). This figure is almost equivalent to Fig. 6, except that the range of allowable temperatures is restricted

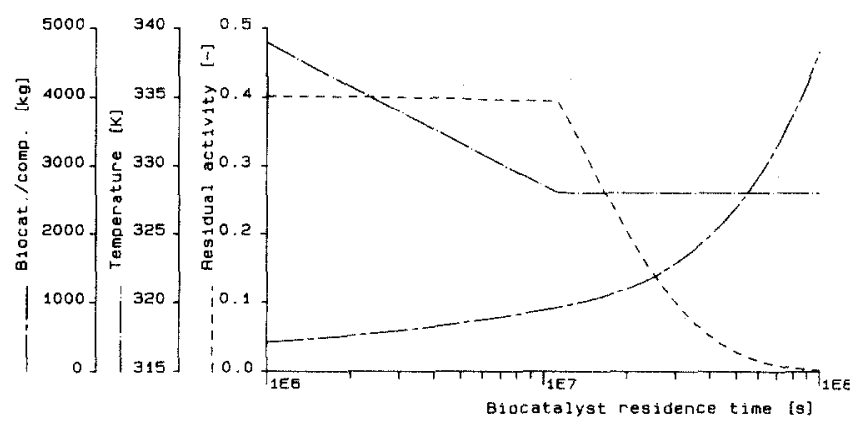

Fig. 7. The same as Fig. 6, but with a minimum operating temperature of $328 \mathrm{~K}$.

by a minimum at $328 \mathrm{~K}$. For biocatalyst residence times longer than approximately $10^{7} \mathrm{~s}$, the reactor temperature is higher than without this restriction. This causes a low residual biocatalyst activity; thus more biocatalyst per compartment is needed to keep the degree of conversion at its specified value.

Having illustrated how to obtain combinations of operating temperature and biocatalyst residence time, the task of determining the economic optimum from these technological suboptima remains. Different choices of biocatalyst residence time resulted in different values of the reactor temperature (in 


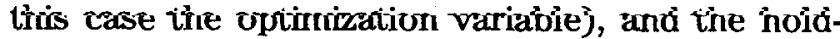
up of biocatalyst. The hold-up of biocatalyst is proportional to the reactor volume; thus it is a measure for the investment-related fixed costs. This can be quantified by the six-tenths rule which is formulated by eqn. (15). The parameter $\sigma_{1}$ stands for the annual investment-related fixed costs. These are estimated as $30 \%$ of the investment costs for a reference reactor. The reactor which was dimensioned for $N_{\mathrm{p}}=1$ in Table 4 is chosen as this reference (biocatalyst hold-up, $6693 \mathrm{~kg} ; N=10 ; H=13.7 \mathrm{~m}$; diameter, $1.21 \mathrm{~m}$ ). The investment costs for this reactor are estimated as US $\$ 500000$; thus $\sigma_{1}$ is approximately US $\$ 150000$ year $^{-1}$. The consumption of biocatalyst is the most important variable cost factor that is changed by different values of the biocatalyst residence time. The biocatalyst cost is given by eqn. (16) where $\sigma_{2}$ is the biocatalyst price (US\$9 $\mathrm{kg}^{-1}$ ). Other cost factors which are incorporated in eqn. (6) are independent of the biocatalyst residence time and do not need to be taken into account when minimizing the total costs function:

investment-related fixed costs

$$
=\sigma_{1} \times\left(\frac{\text { biocatalyst hold-up }}{\text { reference hold-up }}\right)^{0.6}
$$

biocatalyst cost

$$
=\sigma_{2} \times \text { biocatalyst consumption rate }
$$

In Fig. 8, the economically optimal reactor design is determined graphically for these values of the parameters $\sigma_{1}$ and $\sigma_{2}$. The process capacity is 100 ton HFCS day ${ }^{-1}$ and the reactor is assumed to operate at a uniform temperature which should exceed $328 \mathrm{~K}$. The choice of the biocatalyst residence time corresponds to the minimum of the sum of eqns. (15) and (16), and thus of the total cost

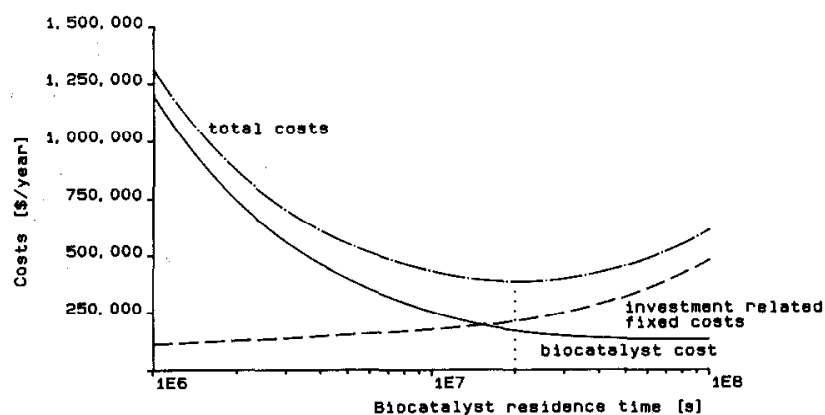

Fig. 8. Determination of the optimal biocatalyst residence time for the HFCS process with a capacity of 100 tons day $^{-1}$ dry weight and a minimum operating temperature of $328 \mathrm{~K}(N=10$; $N_{p}=1 ; \sigma_{1}=\mathrm{US} \$ 150000$ year $^{-1} ; \sigma_{2}=\mathrm{US} \$ 9 \mathrm{~kg}^{-1} ;$ reference holdup, $6693 \mathrm{~kg}$ biocatalyst)
Function. Tine economicafíy optimai situation is characterized by the minimum allowable temperature of $328 \mathrm{~K}$ and a biocatalyst residence time of approximately $2.0 \times 10^{7} \mathrm{~s}( \pm 230$ days $)$. It is noted that this biocatalyst residence time is much longer than the previously mentioned 50 days. Nevertheless, this can hardly be explained by a too conservative estimation of $\sigma_{1}$, as illustrated in Table 5 .

The result obtained by this ecombmic evaluation of the technological suboptima for $N=10$ can be optimized further by taking the number of compartments as an additional optimization variable. Therefore economic optima for a range of reactors need to be compared, taking $\sigma_{1}$ as a function of the total number of compartments. When the influence of the number of compartments in the MFBR on $\sigma_{1}$ is estimated as $\sigma_{1}=\operatorname{US} \$(120000+3000 \mathrm{~N})$ year $^{-4}$, the economically optimal situation as a function of $N$ is given in Table 6 and Fig. 9. For all the process simulations, the optimai temperature equals the minimal allowable temperature. It follows that, from an economic point of view, a reactor with six compartments results in minimization of the total costs for conversion of a glucose syrup to a 42 wt.\% fructose syrup. These costs are only a little more than $2 \%$ of the market price of the syrup produced.

\section{Discussion and conclusions}

The activity and stability of biocatalysts are influenced by environmental parameters. Therefore the reaction conditions contribute significantly to the overall process economy. For reaction systems with continuous refreshment of such a biocatalyst, the process optimization can be split into technical and economic parts. The technical aspect is the determination of the optimal temperature at a given biocatalyst residence time. At this temperature, the biocatalyst productivity is maximal, resulting in a minimization of both the biocatalyst consumption and the hold-up; thus the smallest possible reactor is obtained. Costs functions for biocatalyst and investment-related fixed costs can be used to determine the economic optimum from various possible suboptimal combinations of biocatalyst residence time and operating temperature.

From a study of the glucose isomerase process in an MFBR, it appears that only a small reduction in biocatalyst consumption $( \pm 5 \%)$ can be obtained by imposing a temperature gradient on the reactor. It is not likely that this will be very different for other biocatalysts and it is concluded that, in general, only the use of expensive biocatalysts may justify 
TABLE 5. Influence of $\sigma_{1}$ on optimal process conditions and costs for 100 tons day ${ }^{-1}$ dry weight production of high fructose corn syrup in a multistage fixed-bed reactor $\left(T_{\min }=328 \mathrm{~K} ; N=10 ; N_{\mathrm{p}}=1 ; m=4 ; \epsilon=0.65 ; S_{0}=513 \mathrm{~kg} \mathrm{~m}^{-3} ; \xi=0.4421 ;\right.$ mass flow, $2.571 \mathrm{~kg} \mathrm{~s}^{-1} ; \sigma_{2}=\mathrm{US} \$ 9 \mathrm{~kg}^{-1}$; reference hold-up, $6693 \mathrm{~kg}$ )

\begin{tabular}{|c|c|c|c|c|c|}
\hline $\begin{array}{l}\sigma_{1} \\
\left(\mathrm{US} \$ \text { year }^{-1}\right)\end{array}$ & $\begin{array}{l}\text { Biocatalyst } \\
\text { hold-up in MFBR } \\
(\mathrm{kg})\end{array}$ & $\begin{array}{l}\text { Biocatalyst } \\
\text { residence time } \\
\text { (s) }\end{array}$ & $\begin{array}{l}\text { Investment } \\
\text { related fixed costs } \\
\text { (US } \$ \text { year }^{-1} \text { ) }\end{array}$ & $\begin{array}{l}\text { Biocatalyst } \\
\text { cost } \\
\left(\text { US } \$ \text { year }^{-1} \text { ) }\right.\end{array}$ & $\begin{array}{l}\text { Total costs } \\
\text { (US\$ year }^{-1} \text { ) }\end{array}$ \\
\hline 100000 & $13.5 \times 10^{3}$ & $2.40 \times 10^{7}$ & $152 \times 10^{3}$ & $159 \times 10^{3}$ & $311 \times 10^{3}$ \\
\hline 150000 & $12.0 \times 10^{3}$ & $2.00 \times 10^{7}$ & $213 \times 10^{3}$ & $171 \times 10^{3}$ & $384 \times 10^{3}$ \\
\hline 200000 & $11.5 \times 10^{3}$ & $1.82 \times 10^{7}$ & $276 \times 10^{3}$ & $179 \times 10^{3}$ & $455 \times 10^{3}$ \\
\hline 300000 & $10.5 \times 10^{3}$ & $1.51 \times 10^{7}$ & $393 \times 10^{3}$ & $196 \times 10^{3}$ & $589 \times 10^{3}$ \\
\hline 500000 & $9.5 \times 10^{3}$ & $1.20 \times 10^{7}$ & $618 \times 10^{3}$ & $225 \times 10^{3}$ & $843 \times 10^{3}$ \\
\hline
\end{tabular}

TABLE 6. Influence of $N$ on various process conditions and costs for 100 ton day $^{-1}$ dry weight production of high fructose corn syrup in a multistage fixed-bed reactor $\left(T_{\min }=328 \mathrm{~K}, N_{\mathrm{p}}=1\right.$ $m=4, \epsilon=0.65 ; S_{0}=513 \mathrm{~kg} \mathrm{~m}^{-3} ; \xi=0.4421 ;$ mass flow, 2.571 $\mathrm{kg} \mathrm{s}{ }^{-1} ; \sigma_{2}=\mathrm{US} \$ 9 \mathrm{~kg}^{-1} ; \sigma_{1}=\mathrm{US} \$(120000+3000 \mathrm{~N}) \mathrm{year}^{-1}$ reference hold-up $=6693 \mathrm{~kg}$ )

\begin{tabular}{rllll}
\hline$N$ & $\begin{array}{l}\text { Biocatalyst } \\
\text { hold-up } \\
(\mathrm{kg})\end{array}$ & $\begin{array}{l}\omega_{1} \\
(-)\end{array}$ & $\begin{array}{l}T \\
(\mathrm{~K})\end{array}$ & $\begin{array}{l}\tau_{\text {biocatalyst }} \\
(\mathrm{s})\end{array}$ \\
\hline 1 & $21.4 \times 10^{3}$ & 0.3050 & 328 & $2.64 \times 10^{7}$ \\
2 & $16.1 \times 10^{3}$ & 0.2461 & 328 & $2.31 \times 10^{7}$ \\
3 & $14.5 \times 10^{3}$ & 0.2228 & 328 & $2.26 \times 10^{7}$ \\
4 & $13.7 \times 10^{3}$ & 0.2109 & 328 & $2.21 \times 10^{7}$ \\
5 & $13.3 \times 10^{3}$ & 0.2046 & 328 & $2.17 \times 10^{7}$ \\
6 & $12.9 \times 10^{3}$ & 0.2009 & 328 & $2.13 \times 10^{7}$ \\
7 & $12.7 \times 10^{3}$ & 0.1989 & 328 & $2.11 \times 10^{7}$ \\
8 & $12.5 \times 10^{3}$ & 0.1979 & 328 & $2.08 \times 10^{7}$ \\
9 & $12.3 \times 10^{3}$ & 0.1978 & 328 & $2.06 \times 10^{7}$ \\
10 & $12.2 \times 10^{3}$ & 0.1968 & 328 & $2.05 \times 10^{7}$ \\
11 & $12.1 \times 10^{3}$ & 0.1980 & 328 & $2.02 \times 10^{7}$ \\
12 & $12.0 \times 10^{3}$ & 0.1999 & 328 & $2.00 \times 10^{7}$ \\
\hline
\end{tabular}

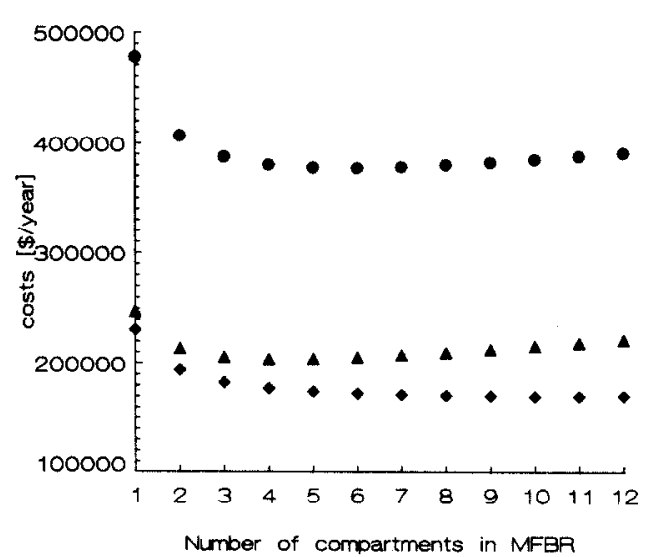

Fig. 9. Minimal costs for operation of an MFBR for the production of HFCS with a capacity of 100 tons day ${ }^{-1}$ dry weight as a function of the number of compartments; $\boldsymbol{\Lambda}$, investment related fixed costs; $\bullet$, biocatalyst costs; $\bullet$, total costs.

the higher investment costs of precise control of a temperature profile over the MFBR. It follows from Fig. 9 that, when taking the costs for $N=1$ as an estimation of the costs for a CSTR, an enzymatic conversion in an MFBR can be carried out approximately $20 \%$ cheaper than in a CSTR. The advantage of the MFBR over the fixed-bed reactor is that, in order to operate the process at a constant capacity and conversion, only one MFBR is needed while at least six fixed-bed reactors are required [3]. According to the sixth-tenths rule, this results in lower investment costs for the MFBR. Furthermore, a fixed-bed reactor requires a cycle of shutdown, reloading with fresh enzyme and start-up while the handling of solids for the MFBR is continuous and automatic. The amounts of enzyme required for the MFBR and a fixed-bed reactor are approximately equal, as shown previously [3]. It is concluded that, compared with the conventional reactor types (CSTR and fixed bed), the use of an MFBR may significantly favour the economics of an enzyme-catalysed reaction step.

However, when making a choice for a reactor type, some practical considerations need to be taken into account. It is, for example, questionable whether the MFBR is an attractive reactor type for the HFCS process. Owing to the good operational stability of the biocatalyst, the residence time of the solids is very long for a continuous process. Furthermore, the residence time of the biocatalyst is long compared with fluctuations in the demand of HFCS, which is relatively small in the winter. It is concluded that the MFBR is not a generally applicable reactor type, but it might be a good alternative for some processes, such as the enzymatic purification of specific industrial waste-water streams [22]. The optimization method presented can be applied generally to design reactors that allow for continuous refreshment of an unstable biocatalyst.

\section{Acknowledgments}

These investigations were supported in part by the Netherlands Technology Foundation (STW) and 
by DSM Central Laboratory (Geleen, Netherlands). Immobilized glucose isomerase from Gist-brocades N.V. (Delft, Netherlands) and IBIS (International Bio-synthetics, Rijswijk, Netherlands) was obtained free of charge.

\section{References}

1 J. Oestergaard and S.L. Knudsen, Starch, 28 (1976) 350. 2 W. Carasik and J.O. Carrol, Food Technol., 37 (1983) 85.

3 H.J. Vos, D.J. Groen, J.J.M. Potters and K.Ch.A.M. Luyben, Biotechnol. Bioeng., 36 (1990) 367.

4 F.H. Verhoff and J.J. Furjanic, Ind. Eng. Chem., Process Design Develop., 22 (1983) 192.

5 H.J. Vos, C. van Houwelingen, M. Zomerdijk and K.Ch.A.M. Luyben, Biotechnol. Bioeng., 36 (1990) 387.

6 L.G. Karlsen and J. Villadsen, Biotechnol. Bioeng., 26 (1984) 1485.

7 L.Y. Ho and A.E. Humphrey, Biotechnol. Bioeng., 12 (1970) 291.

8 C. Kim, H.S. Kim and D.D. Ryu, Biotechnol. Bioeng., 24 (1982) 1889.

9 K.R. Westerterp, W.P.M. van Swaaij and A.A.C.M. Beenackers, Chemical Reactor Design and Operation, Wiley, New York, 1984, pp. 674-688.

10 H.J. Vos, M. Zomerdijk, D.J. Groen and K.Ch.A.M. Luyben, Biotechnol. Bioeng., 36 (1990) 377.

11 A. Sadana, Enzyme Microb. Technol, 4 (1982) 44.

12 J.P. van der Wiel, Continuous Recovery of Bioproducts by Adsorption, Ph.D. Thesis, Delft University of Technology, 1989.

13 H.J. Vos, P.J. Heederik, J.J.M. Potters and K.Ch.A.M. Luyben, Bioprocess Eng., 5 (1990) 63.

14 J.F. Richardson and W.N. Zaki, Trans. Inst. Chem. Eng., 32 (1954) 35.

15 J.A. Roels and R. van Tilburg, ACS Symp. Ser., 106 (1979) 147.

16 Landholt-Bornstein, Zahlenwerte and Functionen, Part II, Vol. 5, 6th edn., Springer, Heidelberg, 1969, p. 376.

17 R.C. Weast (ed.), Handbook of Chemistry and Physics, 67th edn., CRC Press, Boca Raton, FL, 1986, p. D-231.

18 P. Tournié, C. Laguérie and J.P. Couderc, Chem. Eng. Sci., 34 (1979) 1247.

19 J.V. Hupkes and R. van Tilburg, Starch, 28 (1976) 356.

$20 \mathrm{G}$. Beech, Fortran $I V$ in Chemistry, An Introduction to Computer-Assisted Methods, Wiley, London, 1975, pp. $42-47$.

21 IBIS (International Biosynthetics), Rijswijk, Netherlands, personal communication, 1989.

22 A. Wiseman, Topics in Enzyme and Fermentation Technology, Vol. 7, Wiley, New York, 1983, pp. 264-270.

\section{Appendix A: Nomenclature}

$\mathrm{Bi} \quad \mathrm{R}_{\mathrm{p}} k_{\mathrm{L}, \mathrm{s}} / D_{\text {eff, }}$, biot number

$c_{\mathrm{L}}$ dimensionless concentration, defined in Table 1

$\dot{C} \quad$ costs per unit of time
$D_{\text {eff }} \quad$ effective diffusivity $\left(\mathrm{m}^{2} \mathrm{~s}^{-1}\right)$

$\mathrm{Da}_{1}$ Damkohler 1 number, defined in Table 1

$E_{\mathrm{d}}$ deactivation energy for biocatalyst $\left(\mathrm{J} \mathrm{mol}^{-1}\right)$

$E_{\mathrm{r}} \quad$ activation energy for reaction $\left(\mathrm{J} \mathrm{mol}^{-1}\right)$

$H$ expanded bed height in reactor compartment (m)

$k_{\mathrm{d}} \quad$ first-order enzyme deactivation constant $\left(\mathrm{s}^{-1}\right)$

$k_{\mathrm{do}} \quad$ initial first-order deactivation constant $\left(\mathrm{s}^{-1}\right)$

$k_{r} \quad$ general reaction rate constant for fresh biocatalyst

$k_{\mathrm{r}}{ }^{\prime} \quad$ general reaction rate constant (dimensions unspecified)

$k_{0} \quad$ zero order rate constant inside particle ( $\mathrm{mol}$ $\mathrm{m}^{-3} \mathrm{~s}^{-1}$ )

$k_{1} \quad$ first-order rate constant inside particle $\left(\mathrm{s}^{-1}\right)$

$k_{\mathrm{ro}} \quad$ initial value of general reaction rate constant

$K_{\text {eq }} \quad P_{\text {eq }} / S_{\text {eq }}$ equilibrium constant

$K_{\mathrm{M}} \quad$ Michaelis-Menten constant $\left(\mathrm{mol} \mathrm{m}^{-3}\right)$

$m$ number of liquid mixing zones per reactor compartment

$n_{\mathrm{RZ}}$ exponent in the Richardson-Zaki equation

$N$ total number of reactor compartments

$N_{\mathrm{p}} \quad$ number of parameters for temperature polynomial (eqn. (14))

$P \quad$ product concentration $\left(\mathrm{mol} \mathrm{m}^{-3}\right)$

$R$ universal gas constant $\left(\mathrm{J} \mathrm{mol}^{-1} \mathrm{~K}^{-1}\right)$

$\boldsymbol{R}_{\mathrm{p}} \quad$ particle radius (m)

$S_{\text {eq }} \quad$ equilibrium concentration $\left(\mathrm{mol} \mathrm{m} \mathrm{m}^{-3}\right)$

$S_{\mathrm{L}} \quad$ substrate bulk concentration ( $\left(\mathrm{nol} \mathrm{m} \mathrm{m}^{-3}\right.$ )

$S^{0} \quad$ arbitrary substrate concentration $\left(\mathrm{mol} \mathrm{m}^{-3}\right)$

$S_{0} \quad$ substrate inlct concentration $\left(\mathrm{mol} \mathrm{m}{ }^{-3}\right)$

$T$ absolute temperature (K)

$u_{\imath}$ particle free falling velocity $\left(\mathrm{m} \mathrm{s}^{-1}\right)$

$u_{0} \quad$ superficial liquid velocity $\left(\mathrm{m} \mathrm{s}^{-}\right)$

$V_{\max }$ maximum reaction rate inside particle (mol $\mathrm{m}^{-3} \mathrm{~s}^{-1}$ )

\section{Greek letters}

$\epsilon \quad$ liquid fraction in fluidized bed

$\eta_{\text {ov }}$ overall effectiveness factor

$\mu \quad$ viscosity (Pas)

$\nu_{\mathrm{L}} \quad$ dimensionless concentration dependence of the reaction rate for bulk concentrations

$\xi \quad$ conversion

$\rho$ density $\left(\mathrm{kg} \mathrm{m}^{-3}\right)$

$\sigma_{1}$ annual investment related fixed costs (US\$ year $^{-1}$ )

$\sigma_{2} \quad$ biocatalyst price (US\$ $\mathrm{kg}^{-1}$ )

$\tau_{\mathrm{b}} \quad$ biocatalyst residence time in reactor (s)

$\Phi \quad \frac{1}{3} R_{\mathrm{p}} k_{\mathrm{1}}{ }^{1 / 2} / D_{\text {eff, }} \mathrm{s}^{1 / 2}$, first-order reaction modulus

$\omega_{n} \quad$ active fraction of biocatalyst in compartment $n$ 